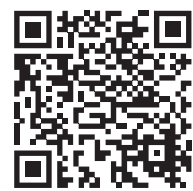

Palabras clave: Cuidados en accidente cerebrovascular,

entrenamiento con simulación, paciente estandarizado, enfoque de seguimiento, entrenamiento interprofesional, entrenamiento de equipos.

Keywords: Stroke care, simulation training, standardized patient, tracking approach, interprofessional training, team training.

* Licenciado en Medicina de la Facultad de Medicina Clínica Alemana Universidad del Desarrollo. Santiago, Chile.

¥ Magíster en Educación Médica. Directora del Núcleo de Simulación Interprofesional. Facultad de Medicina Clínica Alemana Universidad del Desarrollo. Santiago, Chile. $\S$ Licenciado en Medicina. Clínica Alemana de Santiago. Chile.

Recibido: 20/10/2020 Aceptado: 26/11/2020

doi: $10.35366 / 97900$

\section{Simulación multimodal en ACV: paciente estandarizado, simulador de paciente virtual y una aplicación de seguimiento de procesos para el entrenamiento interprofesional}

\author{
Multimodal simulation in stroke: a standardized and virtual \\ patient with mobile tracking app for interprofessional training \\ Victor Navia-González, ${ }^{*, \text { ॠ }}$ Robert Partarrieu-Stegmeier,*,\| \\ Daniela Ahumada-Millar,*,** Soledad Armijo-Rivera, ${ }^{\ddagger, \neq \neq}$ Pablo Lavados $§, \S \S$
}

\section{RESUMEN}

Introducción: El accidente cerebrovascular isquémico es una enfermedad tiempo-dependiente, por lo que el diagnóstico temprano y el manejo interdisciplinario y coordinado entre el equipo prehospitalario y el hospital son fundamentales para el tratamiento. La formación interprofesional ha incorporado diversas tecnologías de enseñanza, como la simulación con pacientes estandarizados, los simuladores virtuales y las aplicaciones telefónicas para el seguimiento de los procesos clínicos. Hay pocas publicaciones sobre la repercusión de la formación multimodal e interprofesional en la gestión inicial del accidente cerebrovascular. El objetivo de este trabajo fue evaluar las repercusiones en la confianza, la percepción de los conocimientos y la satisfacción de los participantes de un taller multimodal para la capacitación interprofesional en el manejo inicial del accidente cerebrovascular. Material y métodos: Se organizó un taller con grupos interprofesionales prehospitalarios e intrahospitalarios, basado en la simulación con paciente estandarizado, la simulación virtual y una aplicación telefónica para el seguimiento de los procesos clínicos. Se aplicó un cuestionario a los 26 participantes para investigar el nivel de satisfacción con la simulación y la aplicación telefónica. Los datos cuantitativos se analizaron utilizando estadísticas descriptivas. Resultados: La tasa de respuesta fue del 67\% (17/26). El 100\% informó una autopercepción de mayor confianza en sus capacidades de manejo del accidente cerebrovascular, el 100\% considera que las instancias de interrogatorio favorecen su aprendizaje y el 93.4\% cree que una aplicación contribuye a mejorar el manejo prehospitalario de los pacientes. Conclusión: Un workshop basado en simulación multimodal y tecnologías para la monitorización de procesos fomenta el proceso de aprendizaje y la confianza del personal capacitado en el manejo inicial de enfermedades tiempo-dependientes como el accidente cerebrovascular.

\section{ABSTRACT}

Introduction: Ischemic stroke is a time-dependent disease, with early diagnosis and interdisciplinary and coordinated management between pre-hospital and in-hospital being has incorporated various teaching technologies, such as simulation with standardized patients, virtual simulators, and telephone applications for tracking clinical processes. There are few publications regarding the impact of multimodal and interprofessional training on initial stroke management. The objective of this work was to evaluate the impact on confidence, perception of knowledge and satisfaction of the participants of a multimodal workshop for interprofessional training in the initial management of stroke. Material and methods: A workshop was organized with pre-hospital and intra-hospital interprofessional groups, based on standardized patient simulation, virtual simulation and a telephone application for tracking clinical processes. A questionnaire was applied to the 26 participants to investigate the level of satisfaction with the simulation and the telephone application. Quantitative data was analyzed using descriptive statistics. Results: The response rate was $67 \%$ (17/26). 100\% reported a self-perception of increased confidence in their stroke management abilities, $100 \%$ feel that debriefing instances favor their learning and $93.4 \%$ believed that an app contributes to the improvement of prehospital management of patients. Conclusion: A workshop based on multimodal simulation and process monitoring technologies promotes the learning process and the confidence of trained personnel in the initial management of time-dependent diseases such as stroke. key to patient management. Interprofessional training

Citar como: Navia-González V, Partarrieu-Stegmeier R, Ahumada-Millar D, Armijo-Rivera S, Lavados P. Simulación multimodal en ACV: paciente estandarizado, simulador de paciente virtual y una aplicación de seguimiento de procesos para el entrenamiento interprofesional. Simulación Clínica. 2020; 2 (3): 99-105. https://dx.doi.org/10.35366/97900 
" ORCID ID

0000-0002-5920-7730.

॥ ORCID ID

0000-0002-9441-6423.

** ORCID ID

0000-0002-7986-0534.

\# ORCID ID

0000-0001-5368-5961.

$\S \S$ ORCID ID

000-0002-9118-9093.

\section{INTRODUCCIÓN}

$E^{\prime}$ I accidente cerebrovascular (ACV) en adultos representa una de las principales causas de mortalidad y discapacidad adquirida en el mundo. $^{1}$

Su tratamiento efectivo es tiempo-dependiente, es decir, los desenlaces clínicos dependen del tiempo desde el inicio de los síntomas hasta la revascularización, ya sea mediante trombólisis endovenosa o trombectomía endovascular. ${ }^{2}$ El diagnóstico precoz y el trabajo en equipo de los profesionales de salud son claves para lograr mejores resultados clínicos con menores complicaciones. ${ }^{3}$

Retrasos en el tratamiento, errores de manejo o malentendidos entre el equipo de salud, son situaciones de riesgo para los pacientes. Un 3-4\% de las complicaciones médicas en casos con ataque cerebrovascular se deben a error humano, y de ellas un $70 \%$ a problemas en la comunicación de los equipos, malos entendidos, mala ejecución de las órdenes o malas decisiones. ${ }^{4}$

Existen múltiples diagramas de flujo para manejar a los sujetos con sospecha de ACV en tiempos óptimos, que cubren diversas estrategias para lograr reducir los tiempos de atención intrahospitalaria, adelantando acciones durante el traslado, pero que no mencionan los medios de entrenamiento utilizados para estos equipos. ${ }^{5}$

Dado el riesgo de entorpecer el tratamiento, patologías graves como el ACV ofrecen menores oportunidades de formación tradicional para profesionales jóvenes, debido a que los docentes deben priorizar la atención clínica por sobre la supervisión de estudiantes $\mathrm{u}$ otros miembros del equipo de salud, dado el natural compromiso ético de los profesionales en dar el tratamiento oportuno a los casos, sin lograr los objetivos docentes. ${ }^{6}$

La simulación y las tecnologías aplicadas a la enseñanza en salud han emergido como herramientas valiosas para el desarrollo de competencias clínicas individuales y de trabajo en equipo, en un ambiente de seguridad psicológica, sin riesgo para los pacientes y con la posibilidad de garantizar acceso y oportunidad de aprendizaje, además de permitir la entrega de retroalimentación específica y la verificación de logros en áreas no observables en el modelo tradicional. ${ }^{7}$

Existen reportes de programas de simulación para mejorar los tiempos de atención en la urgencia que se basan en el uso de pacientes estandarizados ${ }^{8}$ y programas de simulación in situ que han logrado reducir los tiempos intra- hospitalarios a la trombólisis. ${ }^{9}$ En Latinoamérica existen reportes de programas de simulación aplicados en Brasil, que han reducido los tiempos puerta-aguja. ${ }^{10}$

A nivel de formación de pregrado, existen programas de simulación dirigidos a estudiantes de enfermería para el manejo en la urgencia que muestran alta satisfacción con la simulación, ${ }^{11}$ programas de entrenamiento interprofesional basados en el uso de pacientes estandarizados ${ }^{12}$ que se focalizan en la rehabilitación y también cuentan con alta satisfacción de los participantes. En postgrado de neurología, se ha demostrado que luego de la implementación regular de programas de simulación inmersiva para el manejo de códigos de ACV, los tiempos puerta-aguja se redujeron. ${ }^{13}$

El uso de sistemas de comunicación móvil asociados a tracking de movimiento ha sido reportado como un elemento útil para mejorar la coordinación entre profesionales de sistemas prehospitalarios e intrahospitalarios y así reducir el tiempo de atención del ACV desde el inicio de los síntomas. ${ }^{14}$

No encontramos en la literatura descripción de programas de entrenamiento interprofesional usando diversas modalidades de simulación, combinadas con sistemas de comunicación móvil asociados con seguimiento (tracking) de movimiento que se refieran al trabajo conjunto de los niveles de atención prehospitalario y hospitalario.

El siguiente estudio describe la autopercepción de conocimiento, autoconfianza y satisfacción de un grupo interprofesional mediante diversas metodologías con el fin de mejorar el entrenamiento de equipo de salud prehospitalarios e intrahospitalario en el manejo inicial del ACV.

Objetivo: el entrenamiento interprofesional para el manejo del accidente cerebrovascular, mediante simulación virtual, simulación con pacientes estandarizados y una aplicación de seguimiento de procesos clínicos que fomente tanto el aprendizaje como la confianza al momento de la toma de decisiones del personal de salud.

\section{MATERIAL Y MÉTODOS}

Se realizó un estudio con un diseño cuantitativo, observacional y de corte transversal, cuyo objetivo era evaluar la autopercepción de aprendizaje, confianza y satisfacción entre los participantes de una actividad educativa, realizada durante unas jornadas de capacitación en ACV. 
Se elaboró un circuito de seis estaciones para el manejo interprofesional del ACV en el sistema de salud chileno, referidas a la progresión de la atención de un paciente adulto, en los niveles de atención prehospitalario y hospitalario.

Los recursos educativos utilizados para implementar la actividad fueron: simulación con paciente estandarizado, simulación de paciente virtual, usando software Body Interact ${ }^{\circledR}$, simulación virtual de imágenes diagnósticas, usando software simulador de imágenes de TAC ${ }^{\circledR}$, simulación procedimental de preparación de trombolítico y aplicación telefónica para el seguimiento del proceso clínico de manejo inicial de ACV, usando Join $\mathrm{App}^{\circledR}$.

Las estaciones fueron diseñadas para entrenar y evaluar los desempeños de los equipos participantes en las siguientes competencias específicas y recursos educativos (Figura 1):

\section{Simulación manejo del Accidente Cerebro Vascular}

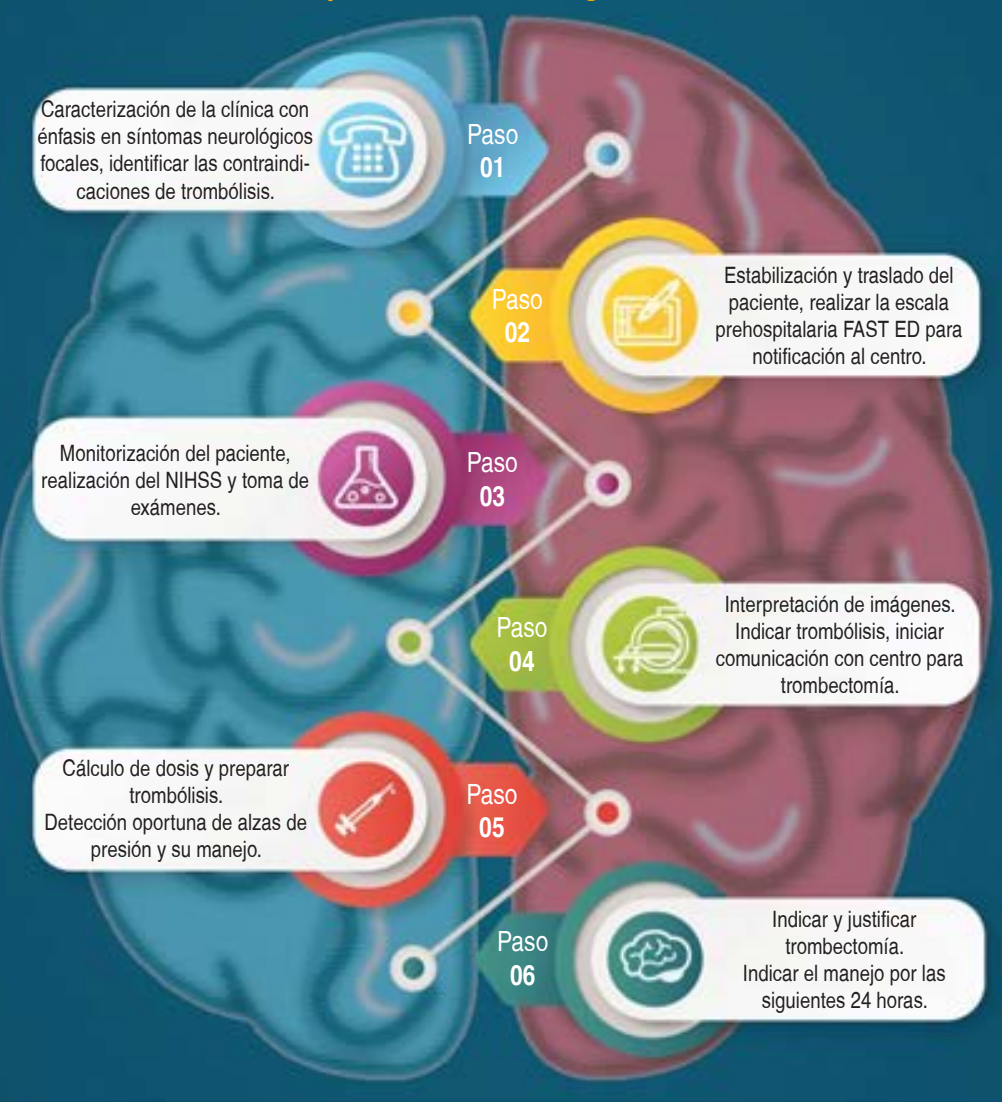

Figura 1:

Organización de workshop

multimodal accidente cardiovascular (ACV).

\section{APLICACIÓN DE TRACKING}

El desarrollo de workshop se apoya en una aplicación de tracking telefónica.
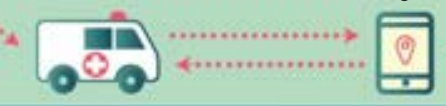
Tabla 1: Evaluación del briefing y escenarios de simulación.

\begin{tabular}{|c|c|c|c|c|c|c|}
\hline \multirow{2}{*}{$\begin{array}{l}\text { Con relación al briefing previo a la simulación } \\
\text { (se obtuvo un total de } 17 \text { respuestas) }\end{array}$} & \multicolumn{2}{|c|}{ Muy de acuerdo } & \multicolumn{2}{|c|}{ De acuerdo } & \multicolumn{2}{|c|}{ No estoy de acuerdo } \\
\hline & Total & $\%$ & Total & $\%$ & Total & $\%$ \\
\hline |Aumentó su autopercepción de confianza & 12 & 70.5 & 5 & 29.4 & 0 & 0 \\
\hline Considera la actividad beneficiosa & 13 & 76.4 & 4 & 23.5 & 0 & 0 \\
\hline \multicolumn{7}{|l|}{$\begin{array}{l}\text { Con relación al briefing posterior a la simulación } \\
\text { (se obtuvo un total de } 17 \text { respuestas) }\end{array}$} \\
\hline Aumentó su sensación de preparación* & 6 & 35.3 & 7 & 41.2 & 1 & 5.8 \\
\hline Aumentó su confianza al tomar decisiones & 10 & 58.8 & 7 & 41.2 & 0 & 0 \\
\hline Desarrolló conocimiento sobre la fisiopatología y farmacología de los casos & 6 & 35.3 & 10 & 53.0 & 1 & 5.8 \\
\hline Desarrolló más confianza al comunicarse con los pacientes & 10 & 58.8 & 7 & 41.2 & 0 & 0 \\
\hline Mejoró sus herramientas de educación con el paciente & 11 & 64.7 & 6 & 35.3 & 0 & 0 \\
\hline $\begin{array}{l}\text { Aumentó su confianza ante la realización de intervenciones que puedan } \\
\text { amenazar la seguridad del paciente }\end{array}$ & 8 & 47.1 & 9 & 52.9 & 0 & 0 \\
\hline
\end{tabular}

* Hay respuestas incompletas en este ítem.

1. Prehospitalario 1: realizar anamnesis dirigida, identificando síntomas neurológicos focales, tiempo de evolución del cuadro clínico, e identificación de contraindicaciones formales de trombólisis. Recursos utilizados: paciente virtual y aplicación de seguimiento.

2. Prehospitalario 2: realizar estabilización y traslado adecuado de paciente notificando a hospitalario con escala prehospitalaria (FAST ED). Recursos utilizados: paciente estandarizado y aplicación de seguimiento.

3. Urgencia hospitalaria 1: realizar monitorización de signos vitales de paciente ingresando a urgencia, realizar puntuación NIHSS (National Institutes of Health Stroke Scale), solicitar exámenes de laboratorio y de imágenes. Recursos utilizados: paciente estandarizado y aplicación de seguimiento.

4. Urgencia hospitalaria 2: interpretar adecuadamente TAC, angioTAC de cerebro, calcular escala ASPECTS (Alberta Stroke Protocol Programme Early CT Score) e identificar sitio de oclusión. Indicar trombólisis, comunicarse con centro con disponibilidad de trombectomía y servicio de ambulancia. Recursos utilizados: simulador de TAC y aplicación de seguimiento.

5. Urgencia hospitalaria 3: calcular dosis, preparar trombólisis, detectar alza tensional y manejarla. Recursos utilizados: simulación de procedimiento de preparación de fármaco y aplicación de seguimiento.
6. Urgencia hospitalaria 4: indicar y justificar trombectomía, dejar indicaciones para primeras 24 horas. Recursos utilizados: paciente estandarizado y aplicación de seguimiento.

La facilitación del taller fue realizada por un equipo interprofesional constituido por enfermeros y otros profesionales del sistema prehospitalario de ambulancias, neurólogos y expertos en simulación y tecnologías educativas.

El taller fue realizado en un periodo de dos horas, distribuidas en: evaluación de conocimientos preintervención; entrega de información inicial de la actividad; organización de los grupos; avance secuencial en las seis estaciones; debriefing final de la actividad; evaluación postintervención y encuesta de satisfacción.

Los participantes fueron distribuidos en seis equipos de trabajo, mediante asignación aleatoria y de acuerdo con sus perfiles profesionales y experiencia clínica. Cada equipo contó con el acompañamiento de un facilitador, quien presentó la situación clínica de la estación asignada y guio la discusión entre los integrantes de cada equipo para mejorar la toma de decisiones. Se dispuso de 15 minutos para resolver cada estación, antes de avanzar a la siguiente.

Los integrantes de los otros equipos se enteraron del estado del paciente y del manejo realizado por el equipo previo mediante el uso de la aplicación de seguimiento.

Cada grupo se reunió posteriormente para un debriefing que fue facilitado por un clínico experto 
en la tarea de la estación con co-debriefing con instructores de simulación.

Se evaluó el conocimiento de los hitos críticos del manejo integral del ACV que requiere trombólisis, usando autorreporte en mediciones pre y postintervención. Se evaluó la satisfacción de los participantes respecto del programa multimodal, de las estaciones de simulación y la aplicación telefónica mediante una escala tipo Likert de tres niveles soportada en plataforma SurveyMonkey. Para el análisis se agruparon las respuestas de tendencia positiva.

\section{RESULTADOS}

El taller contó con un total de 26 participantes, organizados en seis grupos de cuatro o cinco personas cada uno. Diecisiete participantes accedieron voluntariamente a contestar las encuestas de autopercepción y satisfacción, mediante procedimiento de consentimiento informado administrado a través de registro (67\%).

En cuanto a la evaluación de autopercepción de confianza y seguridad de los participantes respecto de la simulación como actividad integrada, tanto en el briefing como en el escenario y el debriefing se obtuvo un alto nivel de acuerdo en todos los ítems de confianza respecto a la metodología utilizada y el manejo de la situación (Tablas 1 y 2).

Los escenarios de simulación permiten aumentar la autoconfianza en la capacidad de toma de decisiones y realización de intervenciones al momento de enfrentarse a pacientes con sospecha de ACV, como lo indica un 100\% de los participantes. Además, permite el desarrollo de habilidades de comunicación y educación hacia los casos, destacado por el $100 \%$ de los participantes (Tabla 1).

Sobre el debriefing, toma relevancia destacar que un $100 \%$ consideró que esta actividad con- tribuyó a su aprendizaje y fue constructiva para su conocimiento (Tabla 2).

En cuanto a la aplicación telefónica de seguimiento o tracking, su uso fue beneficioso al momento del manejo inicial del sujeto con sospecha de ACV. Cabe mencionar que a un $93.4 \%$ le gustó el concepto de la aplicación y cree que contribuye al manejo prehospitalario del ACV, aunque sólo $80 \%$ considera que su uso fue fácil. Además, un $86.7 \%$ la recomendaría como sistema de comunicación prehospitalaria en este tipo de situaciones clínicas (Tabla 3).

\section{DISCUSIÓN}

Este estudio muestra que el entrenamiento multimodal con simulación y una aplicación móvil de tracking posee un efecto beneficioso en la autopercepción de confianza y conocimiento en el grupo entrenado. Como se aprecia en nuestros resultados, esta técnica de enseñanza multimodal fomenta el aprendizaje y confianza al momento de tomar decisiones en patologías tiempodependientes, como es el ACV. El entrenamiento con simulación ha permitido disminuir el tiempo desde el ingreso hospitalario hasta el inicio del tratamiento en los casos con sospecha de ACV en los centros en los que se ha logrado implementar. Un ejemplo de esto es el Hospital Pró-Cardíaco en Río de Janeiro, en el cual el entrenamiento sistematizado interprofesional muestra una reducción significativa del tiempo de entrada a la urgencia hasta el tratamiento inicial, ${ }^{10}$ de manera similar a lo descrito en Noruega por Ajmi. ${ }^{8}$

Por otra parte, es importante destacar el beneficio del entrenamiento interprofesional para disminuir los tiempos de inicio de tratamiento en casos con sospecha de ACV. En nuestra investigación, las estaciones fueron manejadas por grupos con distintos profesionales, logrando mayor semejanza

Tabla 2: Evaluación del debriefing.

\begin{tabular}{|c|c|c|c|c|c|c|}
\hline \multirow{2}{*}{$\begin{array}{l}\text { Con relación al debriefing posterior a la simulación } \\
\text { (se obtuvo un total de } 16 \text { respuestas) }\end{array}$} & \multicolumn{2}{|c|}{ Muy de acuerdo } & \multicolumn{2}{|c|}{ De acuerdo } & \multicolumn{2}{|c|}{ No estoy de acuerdo } \\
\hline & Total & $\%$ & Total & $\%$ & Total & $\%$ \\
\hline La actividad contribuyó a mi aprendizaje & 11 & 68.7 & 5 & 31.2 & 0 & 0 \\
\hline Logró verbalizar sus sentimientos antes de focalizarse en los hechos clínicos & 11 & 68.7 & 4 & 25.0 & 1 & 6.2 \\
\hline Mejoró su juicio clínico & 11 & 68.7 & 4 & 25.0 & 1 & 6.2 \\
\hline Logró reflexionar sobre su propio desempeño & 11 & 68.7 & 5 & 31.2 & 0 & 0 \\
\hline Considera que la simulación fue una evaluación constructiva & 12 & 75.0 & 4 & 25.0 & 0 & 0 \\
\hline
\end{tabular}


Tabla 3: Evaluación de la aplicación de tracking.

\begin{tabular}{|c|c|c|c|c|c|c|}
\hline \multirow{2}{*}{$\begin{array}{l}\text { Uso de aplicación telefónica de tracking (se obtuvo un total de } 16 \text { respuestas) } \\
\text { Con relación al uso de la aplicación JOIN }\end{array}$} & \multicolumn{2}{|c|}{ Muy de acuerdo } & \multicolumn{2}{|c|}{ De acuerdo } & \multicolumn{2}{|c|}{ No estoy de acuerdo } \\
\hline & Total & $\%$ & Total & $\%$ & Total & $\%$ \\
\hline Le gustó el concepto de la aplicación JOIN & 8 & 53.3 & 6 & 40.0 & 1 & 6.6 \\
\hline Su uso me fue fácil* & 6 & 40.0 & 7 & 46.6 & 3 & 20.0 \\
\hline Considera que contribuye al manejo prehospitalario del ataque cerebrovascular* & 10 & 66.6 & 5 & 33.3 & 1 & 6.6 \\
\hline La calidad de imagen fue adecuada* & 11 & 73.3 & 4 & 26.6 & 1 & 6.6 \\
\hline Puede ser utilizada por personal médico y no médico* & 11 & 73.3 & 4 & 26.6 & 1 & 6.6 \\
\hline Le gustaría incorporarla a la práctica diaria & 8 & 53.3 & 6 & 40.0 & 1 & 6.6 \\
\hline La recomendaría como sistema de comunicación prehospitalaria* & 8 & 53.3 & 4 & 26.6 & 2 & 13.3 \\
\hline
\end{tabular}

a la realidad al momento de enfrentarse a este tipo de patologías. La importancia del entrenamiento para el trabajo interprofesional se ve reflejado en un estudio de entrenamiento dirigido sólo para residentes de neurología, esta evidencia y el entrenamiento permiten la reducción de los tiempos de tratamiento cuando los pacientes quedan a cargo de los residentes de neurología, pero se mantienen los tiempos desde que el paciente ingresa al hospital hasta la activación del código de ACV y la toma de imágenes, procesos a cargo del servicio de urgencia que no había sido entrenado. ${ }^{13}$

Por último, es relevante destacar la alta aceptación de los profesionales al enfrentarse a las técnicas de simulación, como se evidencia en nuestros resultados, la totalidad de los participantes considera que la actividad fue beneficiosa para su práctica profesional. El uso de técnicas de simulación es ampliamente utilizado, particularmente en la docencia, donde estudios muestran que estudiantes de enfermería ${ }^{11}$ y de profesiones de salud ${ }^{12}$ han evaluado de forma positiva las simulaciones. La aceptabilidad de una metodología educativa es un punto inicial relevante para provocar aprendizajes, y es la medición viable de obtener en un taller educativo.

Sobre las limitaciones del trabajo, el nivel de medición sólo aborda la satisfacción y autopercepción. Al ser utilizada en un sistema de salud, las mediciones futuras deben enfocarse en la transferencia a la práctica y resultados en pacientes.

\section{CONCLUSIONES}

Este estudio explora la viabilidad de implementar un entrenamiento que articula el trabajo de equipos de prehospitalario e intrahospitalario, siendo en general aceptable para la mayoría de los participantes, lo cual aporta una información relevante y que puede ser de utilidad para mejorar la integración de los diferentes niveles de atención, con el fin de lograr una mejor eficiencia en los procesos y los subsecuentes mejores resultados en pacientes. Esta metodología puede implementarse como programa de educación continua en un sistema en red, dada su factibilidad y aceptabilidad por los participantes usuarios.

\section{REFERENCIAS}

1. GBD 2016 Stroke Collaborators. Global, regional, and national burden of stroke, 1990 to 2016: a systematic analysis for the Global Burden of Disease Study 2016. Lancet Neurol. 2019; 18 (5): 439-458.

2. Saver JL, Goyal M, van der Lugt A, Menon BK, Majoie CB, Dippel DW, et al. Time to treatment with endovascular thrombectomy and outcomes from ischemic stroke: a meta-analysis. JAMA. 2016; 316: 1279-1288.

3. Meretoja A, Keshtkaran M, Saver JL, Tatlisumak T, Parsons MW, Kaste M, et al. Stroke thrombolysis: save a minute, save a day. Stroke. 2014; 45: 1053-1058.

4. Strbian D, Ahmed N, Wahlgren N, Lees KR, Toni D, Roffe C, et al. Trends in door-to-thrombolysis time in the safe implementation of stroke thrombolysis registry: effect of center volume and duration of registry membership. Stroke. 2015; 46: 1275-1280.

5. Meretoja A, Strbian D, Mustanoja S, Tatlisumak T, Lindsberg PJ, Kaste M. Reducing in-hospital delay to 20 minutes in stroke thrombolysis. Neurology. 2012; 79: 306-313.

6. Rall M, Schaedle B, Zieger J, Naef W, Weinlich M. Neue trainingsformen und erhöhung der patientensicherheit. Der Unfallchirurg. 2002; 105: 1033-1042.

7. Motola I, Devine LA, Chung HS, Sullivan JE, Issenberg SB. Simulation in healthcare education: 
a best evidence practical guide. AMEE Guide No. 82. Med Teach. 2013; 35 (10): e1511-e1530. doi: 10.3109/0142159X.2013.818632.

8. Ajmi SC, Advani R, Fjetland L, Kurz KD, Lindner $\mathrm{T}$, Qvindesland SA et al. Reducing door-to-needle times in stroke thrombolysis to $13 \mathrm{~min}$ through protocol revision and simulation training: a quality improvement project in a Norwegian stroke centre. BMJ Qual Saf. 2019; 28 (11): 939-948. doi: 10.1136/ bmjqs-2018-009117.

9. Tahtali D, Bohmann F, Rostek P, Wagner M, Steinmetz $\mathrm{H}$, Pfeilschifter W. Setting up a stroke team algorithm and conducting simulation-based training in the emergency department - a practical guide. J Vis Exp. 2017; (119): 55138. doi: 10.3791/55138.

10. Carvalho VS Jr, Picanço MR, Volschan A, Bezerra DC. Impact of simulation training on a telestroke network. Int J Stroke. 2019; 14 (5): 500-507.

11. Baptista RC, Paiva LA, Gonçalves RF, Oliveira LM, Pereira MF, Martins JC. Satisfaction and gains perceived by nursing students with medium and high-fidelity simulation: A randomized controlled trial. Nurse Educ Today. 2016; 46: 127-132.
12. Pinto C, Possanza A, Karpa K. Examining student perceptions of an inter-institutional interprofessional stroke simulation activity. J Interprof Care. 2018; 32 (3): 391-394. doi: 10.1080/13561820.2017.1405921.

13. Ruff IM, Liberman AL, Caprio FZ, Maas MB, Mendelson SJ, Sorond FA, et al. A resident boot camp for reducing door-to-needle times at academic medical centers. Neurol Clin Pract. 2017; 7 (3): 237-245.

14. Munich SA, Tan LA, Nogueira DM, Keigher KM, Chen $M$, Crowley RW, et al. Mobile real-time tracking of acute stroke patients and instant, secure inter-team communication - the join app. Neurointervention. 2017; 12 (2): 69-76. doi: 10.5469/ neuroint.2017.12.2.69.

Correspondencia:

Dra. Soledad Armijo Rivera

Avenida Las Condes 12438,

Lo Barnechea, Santiago, Chile.

E-mail: soledad.armijo@gmail.com 